\title{
MicroRNA-342 inhibits the progression of glioma by directly targeting PAK4
}

\author{
XIANGHE LU ${ }^{1}$, HAOWEN WANG ${ }^{2}$, ZHIPENG SU ${ }^{1}$, LIN CAI $^{1}$ and WENFENG LI ${ }^{2,3}$ \\ Departments of ${ }^{1}$ Neurosurgery and ${ }^{2}$ Radiation Oncology, The First Affiliated Hospital of Wenzhou Medical University; \\ ${ }^{3}$ Laboratory for Advanced Interdisciplinary Research, Center for Personalized Medicine/Institutes of Translational Medicine, \\ The First Affiliated Hospital of Wenzhou Medical University, Wenzhou, Zhejiang 325000, P.R. China
}

Received January 26, 2017; Accepted June 20, 2017

DOI: $10.3892 /$ or.2017.5783

\begin{abstract}
Glioma is an extremely aggressive and lethal type of brain tumour that originates from glial cells. MicroRNA (miRNA) dysregulation has been implicated in the occurrence and progression of many human cancers, including glioma. Thus, some specific miRNAs are potential therapeutic targets for glioma diagnosis, therapy and prognosis. MicroRNA-342 (miR-342) has been reported to be abnormally expressed in various types of cancer. However, the precise roles of miR-342 in glioma remain unknown. The present study showed that miR-342 is relatively downregulated in glioma tissues and cell lines compared with that in adjacent normal tissues and normal human astrocytes. We observed that low miR-342 expression levels are correlated with advanced WHO grades and low KPS scores of glioma patients. In addition, the results of the functional assays demonstrated that miR-342 overexpression inhibits the proliferation and invasion of glioma cells and induces apoptosis. Further investigation revealed that P21 activated kinases 4 (PAK4) is a direct target of miR-342 in glioma. PAK4 was significantly upregulated in glioma tissues and inversely correlated with miR-342 expression. Moreover, PAK4 knockdown can mimic the effects of miR-342 on glioma cell proliferation, invasion and apoptosis. Notably, restoration of expression of PAK4 reversed the suppressive effects induced by the miR-342 in the glioma cells. The upregulation of miR-342 inactivated the AKT and ERK pathways in glioma. These findings may contribute to the understanding of the molecular mechanism underlying the carcinogenesis and progression of glioma, and to provide novel therapeutic target for the treatment of glioma patients.
\end{abstract}

Correspondence to: Professor Wenfeng Li, Department of Radiation Oncology, The First Affiliated Hospital of Wenzhou Medical University, 2 Fuxue Road, Wenzhou, Zhejiang 325000, P.R. China E-mail: wflee_wz@163.com

Key words: glioma, microRNA-342, P21 activated kinases 4, progression

\section{Introduction}

Glioma, the most common type of malignant tumour in the brain, is an extremely aggressive and lethal type of brain tumour that originates from glial cells. In China, glioma has an annual incidence of 5.26 per 100,000 individuals (1). Gliomas are divided into two groups according to the 2007 World Health Organization (WHO) classification: low-grade (grades I and II) and high-grade (grades III and IV) (2). Despite the advances in radiotherapy and chemotherapy treatments following surgical resection, the prognosis of glioma patients still remains poor $(3,4)$. The median survival of the glioma patients who received comprehensive therapy is $<15$ months (5). Poor glioma prognosis is partially due to the oncogenic nature and rapid growth of glioma cell and local invasion of tumour into normal brain tissues $(6,7)$. Therefore, improving understanding regarding the underlying mechanisms involved in the formation and progression of glioma is essential to the identification of more effective therapeutic strategies for this disease.

MicroRNA (miRNA) is a group of endogenous, singlestranded and non-protein-coding smallRNAs(20-23 nucleotides) without open reading frames (8). miRNAs negatively regulate the expression of their target genes by undergoing base pairing with the 3'-untranslated regions (3'-UTRs) of their target messenger RNAs (mRNAs). The base pairing inhibits mRNA translation or promote mRNA degradation (9). Notably, a single miRNA can modulate several targets simultaneously, whereas a single gene may be regulated by multiple miRNAs (10). Thus, miRNAs are involved in complex regulatory networks that regulate a multitude of biological processes, such as cell proliferation, apoptosis, differentiation, autophagy, angiogenesis, invasion, migration and stem cell renewal (11-13). Moreover, miRNAs are abnormally expressed in various cancers and closely related with cancer initiation and progression (14-16). They also act as tumour suppressors or promoters by directly targeting known oncogenes or tumour suppressor genes (17-19). Therefore, identifying specific miRNAs that play important roles in tumourigenesis and tumour development might provide therapeutic biomarkers for cancer diagnosis, prognosis and therapy.

MicroRNA-342 (miR-342) plays key roles in the development, progression and metastasis of several human cancers 
(20-23). However, the precise roles of miR-342 in glioma remain unknown. Therefore, the aim of our study was to investigate the expression pattern and functions of miR-342 in glioma. Additionally, we examined the molecular mechanisms involved in the association of miR-342 with the proliferation, invasion and apoptosis of glioma cells.

\section{Materials and methods}

Clinical samples. Glioma tissues (49 paired) and corresponding normal adjacent tissues (NATs) were collected from glioma patients undergoing surgical resection at the Department of Neurosurgery, The First Affiliated Hospital of Wenzhou Medical University (Wenzhou, China) from August 2014 to January 2016. None of these glioma patients received prior radiotherapy or chemotherapy. Tissues were frozen in liquid nitrogen immediately after collection and stored at $-80^{\circ} \mathrm{C}$ until further use. This study was approved by the Ethics Committee of The First Affiliated Hospital of Wenzhou Medical University. Written informed consent was also obtained from each patient.

Cell lines, oligonucleotides and cell transfection. Glioma cell lines U251, U87, A172, and LN229 were purchased from American Type Culture Collection (Manassas, VA, USA), and routinely cultured in Dulbecco's modified Eagle's medium (DMEM, Invitrogen, Carlsbad, CA, USA) supplemented with $100 \mathrm{U} / \mathrm{ml}$ penicillin and $100 \mathrm{mg} / \mathrm{ml}$ streptomycin (Invitrogen) and $10 \%$ fetal bovine serum (FBS; Invitrogen). Normal human astrocytes (NHAs) were obtained from ScienCell Research Laboratories (Carlsbad, CA, USA) and grown in astrocyte medium (ScienCell Research Laboratories). All cells were maintained at $37^{\circ} \mathrm{C}$ in a humidified atmosphere containing $5 \% \mathrm{CO}_{2}$.

miR-342 mimics, corresponding miRNA negative control (miR-NC), PAK4-targeted small interfering RNA (PAK4 siRNA) and siRNA negative control (NC siRNA) were chemically synthesized by GenePharma Co., Ltd. (Shanghai, China). PAK4 overexpressed plasmid (pcDNA3.1-PAK4) and blank plasmid (pcDNA3.1) were purchased from Chinese Academy of Sciences (Changchun, China). For in vitro function assays, cells were seeded in 6-well plates at 50-70\% confluence. Transfection and co-transfection was performed through the use of Opti-MEM and Lipofectamine 2000 (Invitrogen) according to the manufacturer's protocol. After 6-h transfection, the culture medium was removed and placed in DMEM containing $10 \%$ FBS.

Reverse transcription-quantitative polymerase chain reaction $(R T-q P C R)$. Total RNA from tissues or cells was extracted using TRIzol reagent (Invitrogen) according to the manufacturer's protocol. The quality and concentration of total RNA was examined using a NanoDrop ${ }^{\circledR}$ ND-1000 spectrophotometer. TaqMan ${ }^{\circledR}$ microRNA assays (Applied Biosystems, Foster City, CA, USA) was used to detect miR-342 expression, with RUN6B as an internal control. To quantify PAK4 mRNA, M-MLV Reverse Transcription system (Promega Corp., Madison, WI, USA) was used to synthesis single-stranded cDNA and qPCR was conducted using SYBR Premix Ex Taq (Takara, Dalian, China), with $\beta$-actin as an internal control.
The primers used in this study were as follows: miR-342 forward, 5'-GTGCTATCTGTGATTGAGGGA'-3 and reverse, 5'-CGGGTGCGATTTCTGTG'-3; RUN6B forward, 5'-CTCG CTTCGGCAGCACA-3' and reverse, 5'-AACGCTTCACGAA TTTGCGT-3'. PAK4 forward, 5'-TCCCCCTGAGCCATT GTG-3' and reverse, 5'-TGACCTGTCTCCCCATCCA-3'; $\beta$-actin forward, 5'-ATGGGTCAGAAGGATTCCTATGTG-3', and reverse, 5'-CTTCATGAGGTAGTCAGTCAGGTC-3' Relative expression was determined using the $2^{-\Delta \Delta \mathrm{Ct}}$ method (24).

3-(4, 5-dimethylthiazol-2-yl)-2, 5-diphenyltetra-zolium bromide (MTT) assays. Briefly, cells were harvested and seeded in 96-well plates at a density of $3 \times 10^{3}$ cells/well. The following day, miR-342 mimics, miR-NC, PAK4 siRNA, NC siRNA, pcDNA3.1-PAK4 or pcDNA3.1 was transfected into cells using Opti-MEM and Lipofectamine 2000. The culture medium was replaced by DMEM containing 10\% FBS at $6 \mathrm{~h}$ post-transfection and then incubated at $37^{\circ} \mathrm{C}$ in a humidified atmosphere containing $5 \% \mathrm{CO}_{2}$ for $0,24,48$ and $72 \mathrm{~h}$. MTT solution $(10 \mu \mathrm{l})(5 \mathrm{mg} / \mathrm{ml}$; Sigma-Aldrich, St. Louis, MO, USA) was added into each well and the plates were incubated at $37^{\circ} \mathrm{C}$ for another $4 \mathrm{~h}$. Subsequently, the culture medium was removed carefully and $150 \mu 1$ dimethyl sulfoxide (SigmaAldrich) was added into each well to solubilize the MTT formazan. The absorbance was measured at a wavelength of $490 \mathrm{~nm}$ with a microplate spectrophotometer (Molecular Devices, Sunnyvale, CA, USA). All experiments were performed in triplicate and repeated three times.

Cell invasion assay. Transfected cells were harvested at $48 \mathrm{~h}$ post-transfection and suspended in FBS-free DMEM. Cells $\left(5 \times 10^{4}\right)$ were placed on the top chambers of 24-well Transwell plates (Corning Inc., Corning, NY, USA) coated with Matrigel (BD Biosciences, Franklin Lakes, NJ, USA). DMEM supplemented with $20 \%$ FBS was used as a chemoattractant in the lower chambers. After $48 \mathrm{~h}$ of incubation at $37^{\circ} \mathrm{C}$ with $5 \% \mathrm{CO}_{2}$, the non-invading cells were removed using a cotton swab. The invasive cells were fixed with $100 \%$ methanol for $10 \mathrm{~min}$, stained with $0.1 \%$ crystal violet for $20 \mathrm{~min}$ and photographed at x200 magnification. The number of invasive cells in five random fields was counted under an inverted microscope (CKX41; Olympus Corp., Tokyo, Japan). All experiments were performed in triplicate and repeated three times.

Flow cytometry analysis. Cell apoptosis was assessed $48 \mathrm{~h}$ after transfection. Transfected cells were harvested with trypsinization, washed in ice-cold PBS and fixed in $80 \%$ ice-cold ethanol in PBS. Subsequently, cells were re-suspended in $1 \mathrm{X}$ binding buffer to a concentration of $1 \times 10^{4}$ cells. Annexin V-FITC apoptosis detection kit (Invitrogen Corp.) was utilized to examine cell apoptosis according to the manufacturer's protocol. Briefly, cells were stained with FITC-Annexin V and propidium iodide (PI). After incubation at room temperature in the dark for $15 \mathrm{~min}$, cell apoptosis was quantified using flow cytometry within $1 \mathrm{~h}$ of staining.

Bioinformatic analysis and luciferase reporter assay. TargetScan (http://www.Targetscan.org/) and PicTar (http:// pictar.mdcberlin.de/) were utilized to predicate the potential target genes of miR-342. The wild-type 3'-UTR segment of the 
A

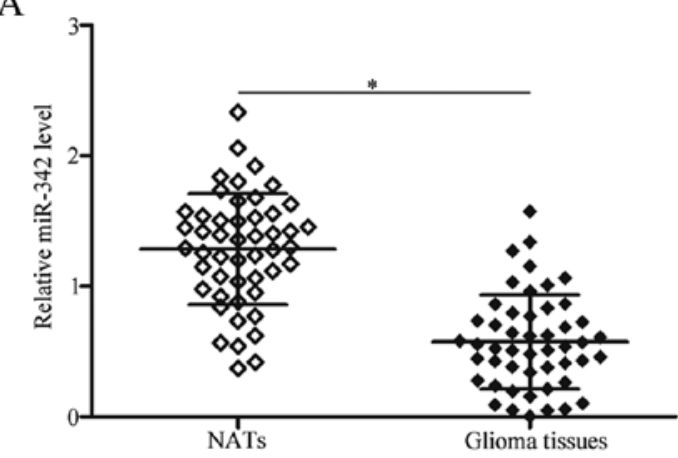

B

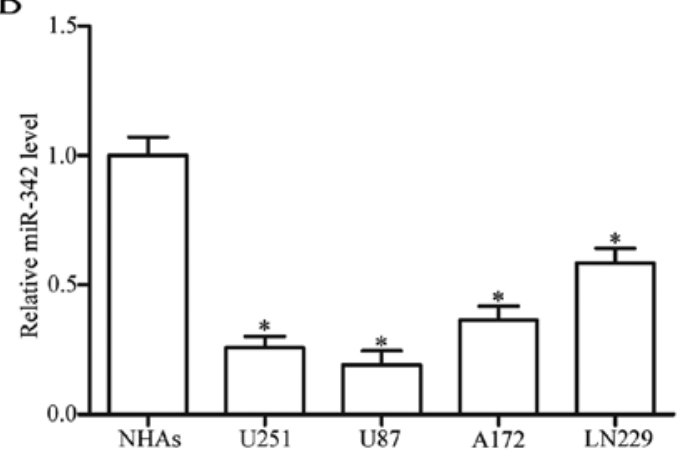

Figure 1. miR-342 is reduced in glioma tissues and cell lines. (A) Expression level of miR-342 in glioma tissues and NATs was examined by RT-qPCR. ${ }^{*} \mathrm{P}<0.05$ compared with NATs. (B) miR-342 expression was determined in five glioma cell lines (U251, U87, A172 and LN229) and normal human astrocytes (NHAs). ${ }^{*} \mathrm{P}<0.05$ compared with NHAs.

PAK4 mRNA containing miR-342 binding sites was amplified and inserted into pMIR Reporter (Ambion, Austin, TX, USA) and named pMIR-PAK4-3'-UTR-Wt. To mutate the binding site of miR-342, its complementary sequence in the 3'UTR of PAK4 (GUGUGAU) was replaced by CACACUC, inserted into pMIR Reporter and named as pMIR-PAK4-3'-UTR-Mut. For the luciferase reporter assay, miR-342 mimics or miR-NC was transfected into cells, along with pMIR-PAK4-3'-UTRWt or pMIR-PAK4-3'-UTR-Mut, using Lipofectamine 2000 according to the manufacturer's protocol. Luciferase activity was determined at 48 -h post-transfection using a DualLuciferase Reporter assay system (Promega), according to the manufacturer's instructions. Renilla luciferase activity was used for normalization.

Western blot analysis. Total proteins were extracted from tissues and cells using the RIPA lysis buffer with protease inhibitors (Roche Diagnostics, Basel, Switzerland) and phosphatase inhibitors (Merck KGaA, Darmstadt, Germany). The concentration of total proteins was quantified using a BCA Protein assay kit (Pierce Biotechnology, Rockford, IL, USA). The same amount of protein was separated by $10 \%$ SDS-PAGE gel electrophoresis, transferred to polyvinylidene difluoride membranes (EMD Millipore, Billerica, MA, USA) and blocked with 5\% fat-free milk. The membranes were then incubated overnight at $4^{\circ} \mathrm{C}$ with primary antibodies: mouse anti-human monoclonal PAK4 (sc-390507; 1:1,000 dilution; Santa Cruz Biotechnology, CA, USA), mouse antihuman monoclonal p-AKT (sc-271966; 1:1,000 dilution; Santa Cruz Biotechnology), mouse anti-human monoclonal AKT
Table I. Association between microRNA-342 expression and clinicopathological factors of glioma patients.

\begin{tabular}{|c|c|c|c|c|}
\hline \multirow[b]{2}{*}{$\begin{array}{l}\text { Clinicopathological } \\
\text { factors }\end{array}$} & \multirow[b]{2}{*}{ Cases } & \multicolumn{2}{|c|}{$\begin{array}{c}\text { microRNA-342 } \\
\text { expression }\end{array}$} & \multirow[b]{2}{*}{ P-value } \\
\hline & & Low & High & \\
\hline Sex & & & & 0.409 \\
\hline Male & 32 & 19 & 13 & \\
\hline Female & 17 & 8 & 9 & \\
\hline Age, years & & & & 0.740 \\
\hline$<55$ & 21 & 11 & 10 & \\
\hline$\geq 55$ & 28 & 16 & 12 & \\
\hline Tumour size, $\mathrm{cm}$ & & & & 0.647 \\
\hline$<5$ & 34 & 18 & 16 & \\
\hline$\geq 5$ & 15 & 9 & 6 & \\
\hline KPS score & & & & 0.035 \\
\hline$<80$ & 26 & 18 & 8 & \\
\hline$\geq 80$ & 23 & 9 & 14 & \\
\hline WHO grade & & & & 0.001 \\
\hline I-II & 23 & 7 & 16 & \\
\hline III-IV & 26 & 20 & 6 & \\
\hline
\end{tabular}

WHO, World Health Organization; KPS, Karnofsky performance score.

(sc-81434; 1:1,000 dilution; Santa Cruz Biotechnology), mouse anti-human monoclonal p-ERK (sc-81492; 1:1,000 dilution; Santa Cruz Biotechnology), mouse anti-human monoclonal ERK (sc-514302; 1:1,000 dilution; Santa Cruz Biotechnology), and mouse anti-human monoclonal GAPDH antibody (sc-47724; 1:1,000 dilution; Santa Cruz Biotechnology). After washing three times with Tris-buffered saline with 0.5\% Tween-20 (TBST; Beyotime Institute of Biotechnology, Haimen, China), the membranes were probed with goat anti-mouse horseradish peroxidase-conjugated secondary antibody (sc-2005; 1:5,000 dilution; Santa Cruz Biotechnology) at room temperature for $1 \mathrm{~h}$. The protein blots were visualized using the ECL Protein Detection kit (Pierce Biotechnology). GAPDH was used as a loading control.

Statistical analysis. The data in this study are presented as mean \pm SD. Data were compared using Student's t-test or one-way ANOVA with SPSS 16.0 software (SPSS, Chicago, IL, USA). The association between miR-342 and clinicopathologic features of glioma was evaluated using Pearson's $\chi^{2}$ test. Differences were considered significant at $\mathrm{P}<0.05$.

\section{Results}

miR-342 is downregulated in glioma tissues and cell lines. To investigate the role of miR-342 in glioma, we measured miR-342 expression levels in 49 paired glioma tissues and NATs. The RT-qPCR results showed that miR-342 expression was significantly lower in glioma tissues than in NATs (Fig. 1A, P<0.05). We further evaluated the association between 
A

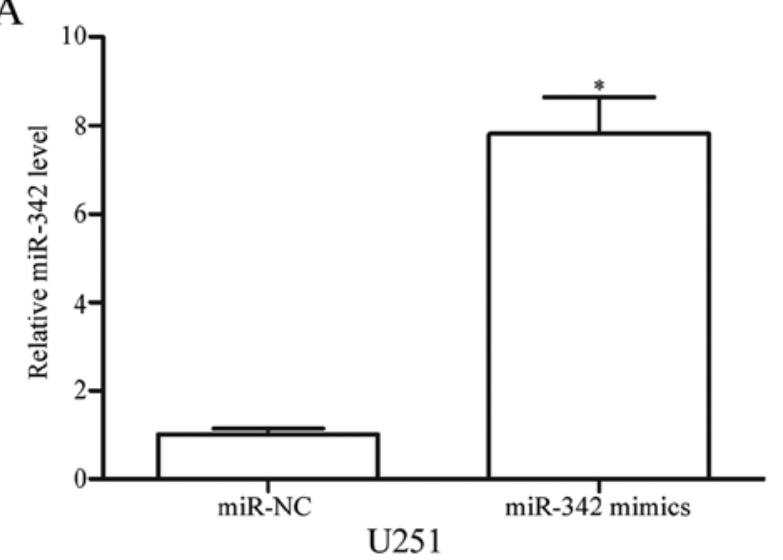

$\mathrm{C}$

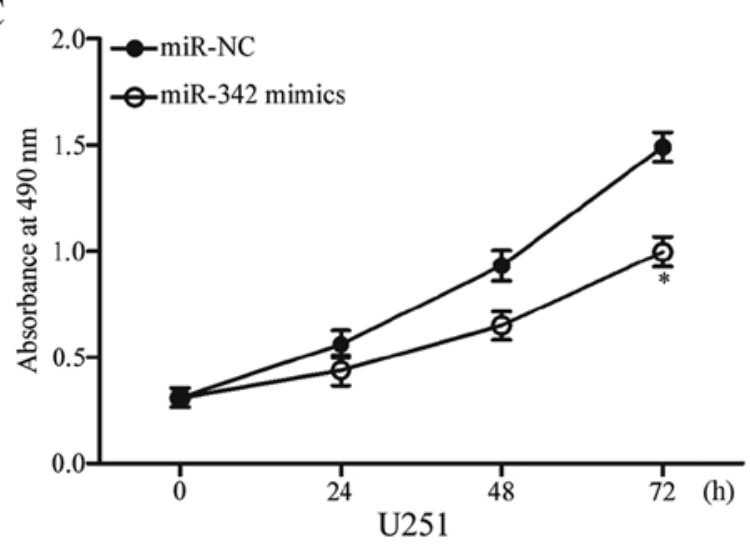

$\mathrm{E}$

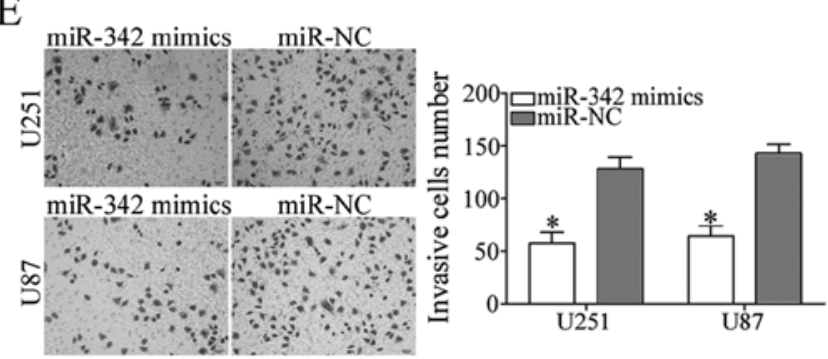

B

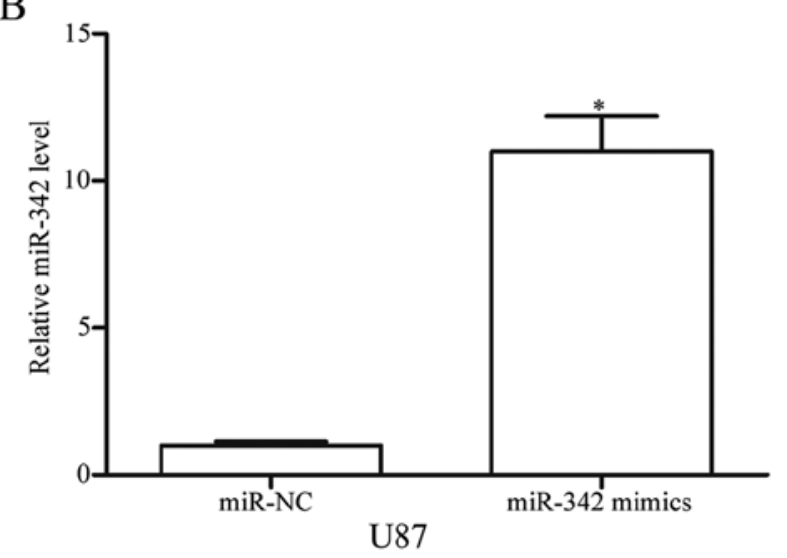

$\mathrm{D}$

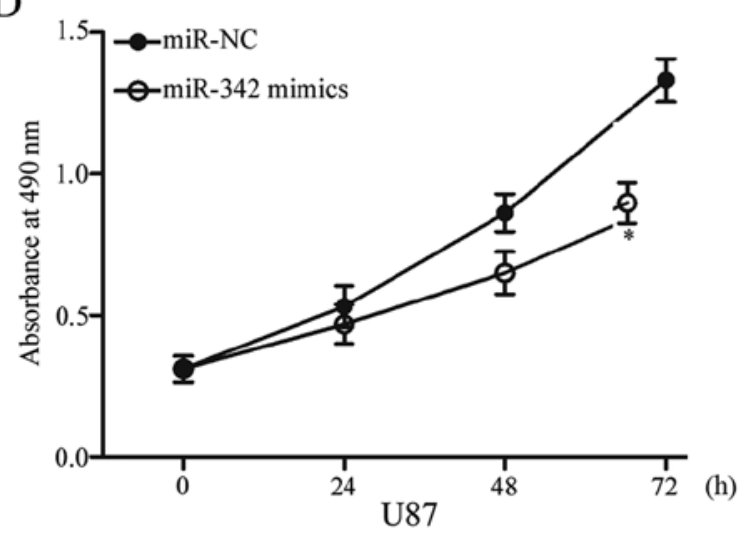

$\mathrm{F}$

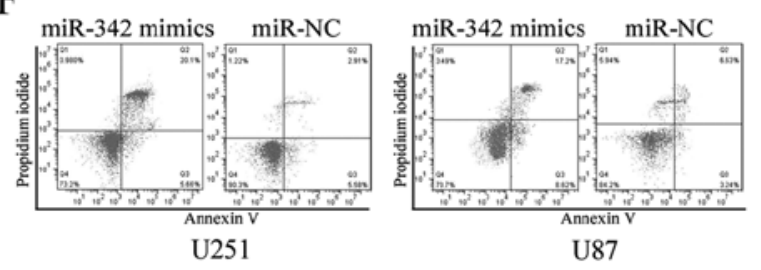

$\square$ miR-342 mimics

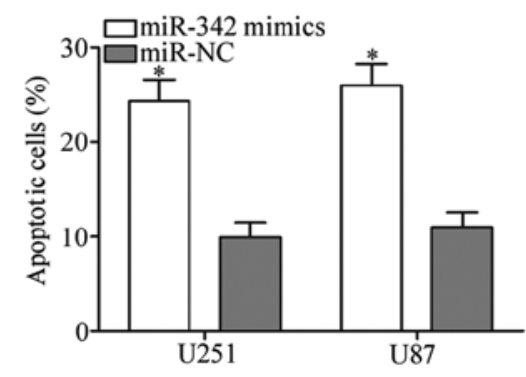

Figure 2. Upregulation of miR-342 inhibits cell proliferation and invasion and induces apoptosis in U251 and U87 cells. (A and B) U251 and U87 cells were treated with miR-342 mimics or miR-NC. RT-qPCR was performed to assess its transfection efficiency. ${ }^{*} \mathrm{P}<0.05$ compared with miR-NC. (C and D) Cell proliferation in the miR-342 mimics and miR-NC group was evaluated by MTT assay. ${ }^{*}<0.05$ compared with miR-NC. (E) Cell invasion abilities of U251 and U87 cells transfected with miR-342 mimics were detected by cell invasion assay. ${ }^{*} \mathrm{P}<0.05$ compared with miR-NC. (F) Apoptosis rate of the U251 and U87 cells transfected with miR-342 mimics or miR-NC was assessed by flow cytometry analysis. "P<0.05 compared with miR-NC.

miR-342 expression and clinicopathological factors of glioma patients. We found that the expression levels of miR-342 were strongly correlated with advanced WHO grades $(\mathrm{P}=0.001)$ and low KPS scores $(\mathrm{P}=0.035)$. No significant association was observed between miR-342 and each of the following parameters: sex, age and tumour size (all $\mathrm{P}>0.05$; Table I). In addition, the expression levels of miR-342 in four glioma cell lines
(U251, U87, A172 and LN229) and normal human astrocytes (NHAs) were examined. As shown in Fig. 1B, expression level of miR-342 decreased in the glioma cell lines in comparison with that in NHAs $(\mathrm{P}<0.05)$. These results suggested that low miR-342 expression is correlated with advanced malignancy of gliomas, and miR-342 may serve as a tumour suppressor in glioma occurrence and progression. 
A

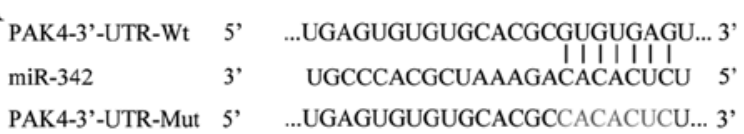

B

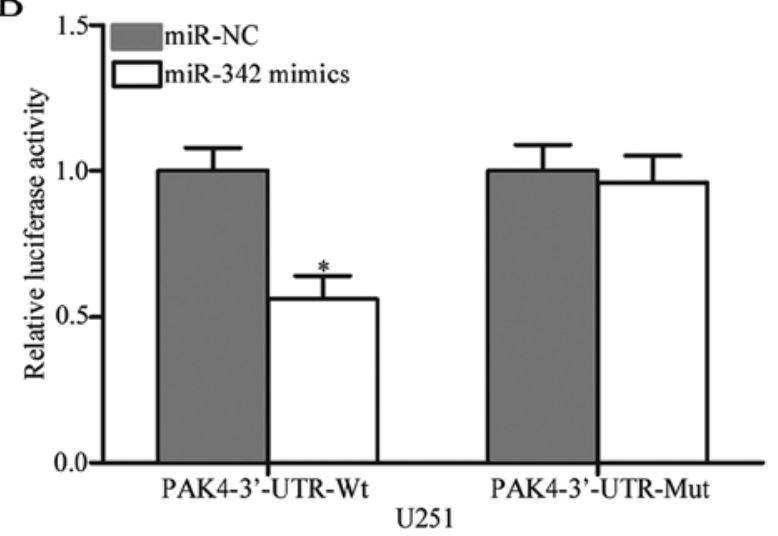

$\mathrm{C}$

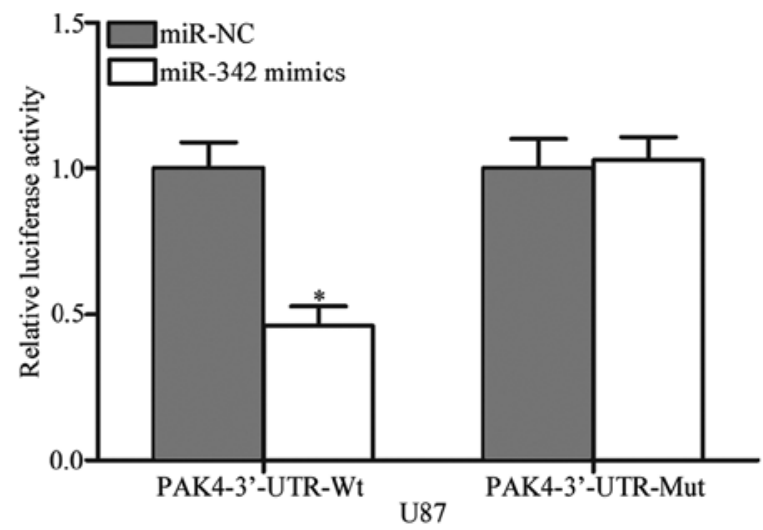

$\mathrm{D}$

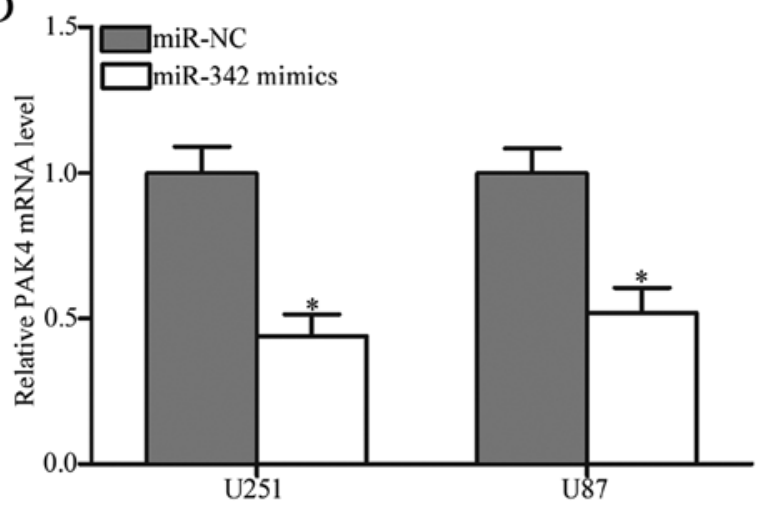

$\mathrm{E}$
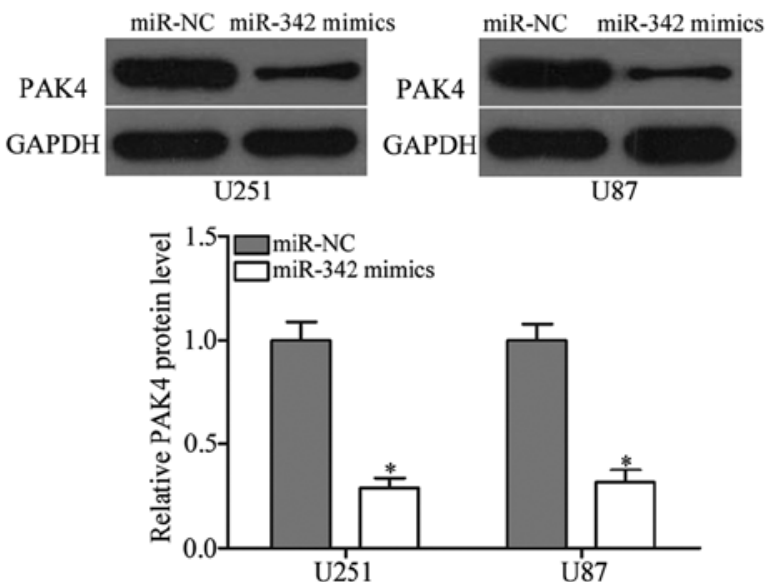

Figure 3. PAK4 is a direct target of miR-342 in glioma. (A) Putative seed-matching sites or mutant sites between miR-342 and 3'-UTR of PAK4 gene. (B and C) Analysis of luciferase activity in the U251 and U87 cells co-transfected with miR-342 mimic or miR-NC, and pMIR-PAK4-3'-UTR-Wt or pMIR-PAK4-3'UTR-Mut. Firefly luciferase activity was normalized to Renilla luciferase activity. ${ }^{*} \mathrm{P}<0.05$ compared with miR-NC. (D) RT-qPCR analysis of PAK4 mRNA level in the U251 and U87 cells transfected with miR-342 mimics or miR-NC. "P<0.05 compared with miR-NC. (E) Western blot analysis was conducted to measure PAK4 protein expression in the U251 and U87 cells transfected with miR-342 mimics or miR-NC. ${ }^{*} \mathrm{P}<0.05$ compared with miR-NC.

miR-342 overexpression inhibits cell proliferation and invasion and activates cell apoptosis in glioma. To investigate miR-342 functions in glioma, we transfected miR-342 mimics into the U251 and U87 cells, which have the lowest miR-342 expression level, to increase its endogenous expression level (Fig. 2A and B, P<0.05). MTT assay was then used to determine the effect of miR-342 overexpression on the cell proliferation of glioma. As shown in Fig. 2C and D, the proliferation of the U251 and U87 cells was suppressed in the group transfected with the miR-342 mimics compared with that in the miR-NC group $(\mathrm{P}<0.05)$. We then utilized cell invasion assay to examine the effect of miR-342 on glioma cell invasion. The results indicated that the U251 and U87 cells with high miR-342 level showed lower invasion capacities than the cells with miR-NC (Fig. 2E, P<0.05). Next, flow cytometry analysis was performed to explore the role of miR-342 in glioma cell apoptosis. As shown in Fig. 2F, the upregulation of miR-342 improved apoptosis in the U251 and U87 cells considerably
$(\mathrm{P}<0.05)$. These results highlighted that miR-342 plays tumoursuppressive roles in glioma by inhibiting cell proliferation and invasion and inducing cell apoptosis. Overall, these results indicated that miR-342 affects the growth, metastasis and apoptosis of glioma cells.

miR-342 directly targets and inhibits PAK4 expression in glioma. After observing the tumour-suppressing roles of miR-342 in glioma, we explored its potential targets using bioinformatics analysis. The results indicated a large number of candidate targets of miR-342. Of these targets, P21 activated kinases 4 (PAK4) was selected for further target identification (Fig. 3A), because it was upregulated in glioma tissues and involved in glioma formation and progression (25). To test this hypothesis, we conducted luciferase reporter assay to validate the interaction between miR-342 and the predicted binding site in the 3'-UTR of the PAK4 gene. As illustrated in Fig. 3B and $\mathrm{C}$, the restoration of miR-342 expression inhibited the 
A

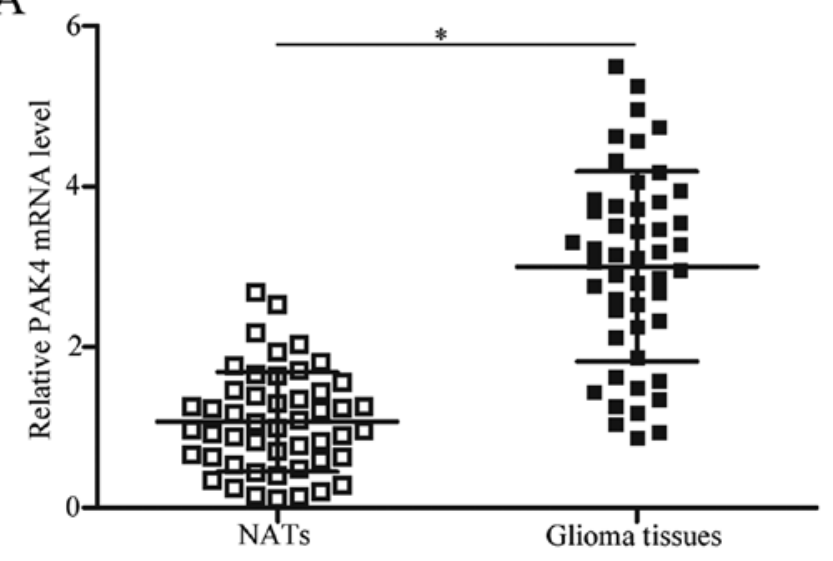

B
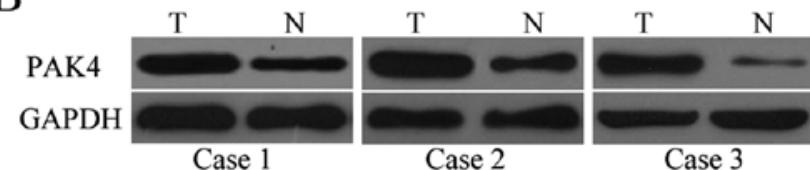

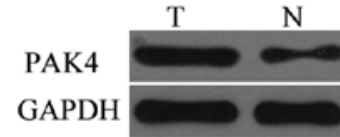

Case 4

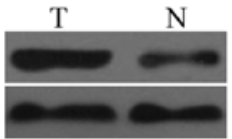

Case 5

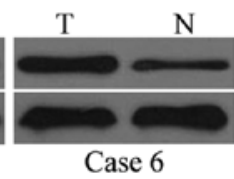

C

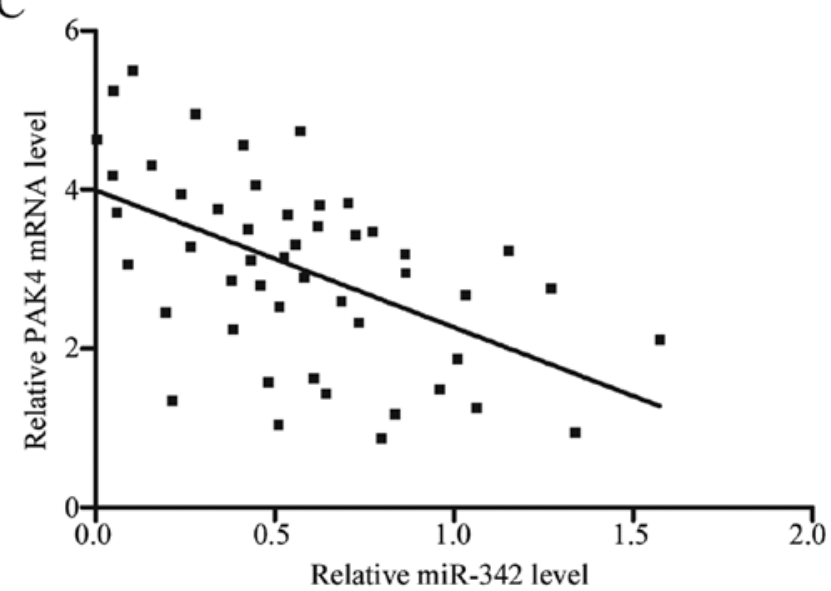

Figure 4. PAK4 expression is upregulated and negatively correlated with miR-342 expression in glioma tissues. (A) The expression levels of PAK4 mRNA in glioma tissues and NATs were determined by RT-qPCR analysis. ${ }^{*} \mathrm{P}<0.05$ compared with NATs. (B) Western blot analysis of PAK4 protein expression in glioma tissues and NATs. T, glioma tissue; N, NAT. (C) Spearman's correlation analysis was utilized to evaluate the correlation between miR-342 and PAK4 mRNA expression in glioma specimens.

luciferase activity of pMIR-PAK4-3'-UTR-Wt $(\mathrm{P}<0.05)$, but not of pMIR-PAK4-3'-UTR-Mut, suggesting that miR-342 specifically targeted the 3'-UTR of PAK4. RT-qPCR and western blot analysis were then performed to detect PAK4 mRNA and protein expression in the U251 and U87 cells transfected with the miR-342 mimics or miR-NC. Our findings revealed that, at the mRNA and protein levels, the PAK4 expression levels decreased in the U251 and U87 cells transfected with the miR-342 mimics compared with that in the miR-NC group (Fig. 3D and $\mathrm{E}, \mathrm{P}<0.05$ ). These data demonstrated that
miR-342 is a post-transcriptional regulator of PAK4 and binds directly to the 3'UTR of the PAK4 gene.

PAK4 expression is upregulated and inversely correlates with miR-342 expression in glioma tissues. To confirm the association between miR-342 and PAK4, we calculated PAK4 mRNA and protein expression levels in glioma tissues and NATs. The data obtained from RT-qPCR and western blot analysis showed that PAK4 mRNA and protein were significantly upregulated in glioma tissues relative to those with NATs (Fig. 4A and B, $\mathrm{P}<0.05)$. Additionally, Spearman's correlation analysis results confirmed that the negative association between PAK4 mRNA expression and miR-342 in glioma tissues (Fig. 4C; $r=-0.5261$, $\mathrm{P}=0.0001)$.

PAK4 downregulation imitates the roles of miR-342 in glioma. PAK4 was identified as a direct target of miR-342 in glioma. We hypothesized that the roles of PAK4 knockdown on glioma cells are similar with those induced by miR-342 overexpression. To confirm this hypothesis, we transfected the U251 and U87 cells with PAK4 siRNA or NC siRNA. As expected, the PAK4 expression decreased in the U251 and U87 cells after transfection with PAK4 siRNA (Fig. 5A, P<0.05). The results of the subsequent functional assays showed that downregulation of PAK4 repressed the proliferation (Fig. 5B and $\mathrm{C}, \mathrm{P}<0.05)$ and invasion $($ Fig. 5D, $\mathrm{P}<0.05)$ and induced apoptosis (Fig. 5E, $\mathrm{P}<0.05$ ) in the U251 and U87 cells. This result supported the hypothesis that PAK4 underexpression plays the same role as that of miR-342 mimics in glioma cells. These results further demonstrated that PAK4 may be a functional target of miR-342 in glioma.

PAK4 reverses the tumour-suppressing effects of miR-342 on glioma cells. Rescue experiments were performed to evaluate the contribution of PAK4 to the roles of miR-342 in glioma. After introducing pcDNA3.1-PAK4 or pcDNA3.1 into the U251 and U87 cells, we performed western blot analysis $72 \mathrm{~h}$ post-transfection. The results showed that PAK4 was upregulated in the pcDNA3.1-PAK4-transfected U251 and U87 cells. In addition, PAK4 expression was recovered in the miR-342 mimic-transfected cells after being transfected with pcDNA3.1-PAK4 (Fig. 6A, P<0.05). The results of the functional rescue experiments showed that reintroduction of PAK4 effectively rescued the effects of miR-342 overexpression on the proliferation (Fig. 6B and $\mathrm{C}, \mathrm{P}<0.05$ ), invasion (Fig. 6D, $\mathrm{P}<0.05$ ) and apoptosis (Fig. 6E, $\mathrm{P}<0.05$ ) of the $\mathrm{U} 251$ and $\mathrm{U} 87$ cells. These data showed clearly that miR-342 exerted tumoursuppressive roles in glioma, at least in part, by suppressing PAK4.

miR-342 is associated with the AKT and ERK pathway in glioma. Previous studies reported that PAK4 plays essential roles in the AKT and ERK pathways $(26,27)$. Thus, to assess the function of miR-342 on these pathways, we transfected miR-342 mimics or miR-NC into the U251 and U87 cells. As shown in Fig. 7, miR-342 overexpression reduced both the p-AKT and p-ERK expression levels in the U251 and U87 cells $(\mathrm{P}<0.05)$. However, it did not affect the entire AKT and ERK expression. These results illustrated that miR-342 can inactivate the AKT and ERK pathways in glioma. 
A

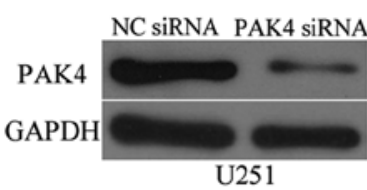

\section{PAK4 GAPDH} $\mathrm{U} 25$

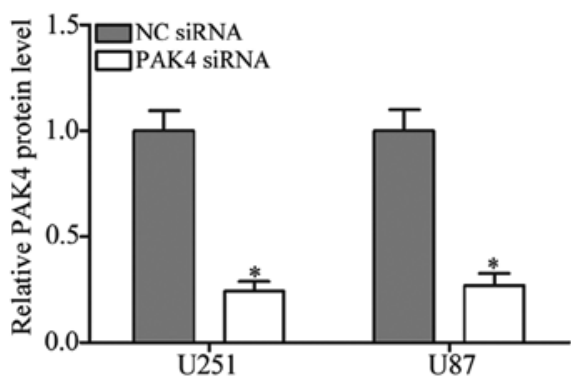

C

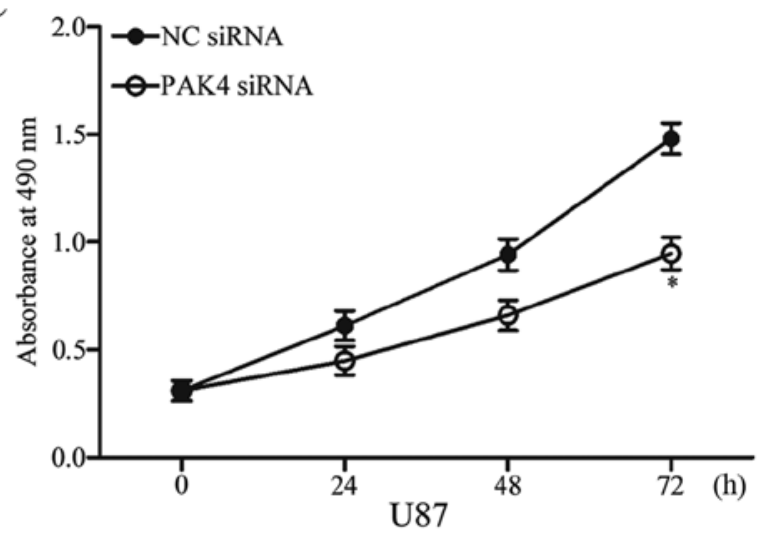

B

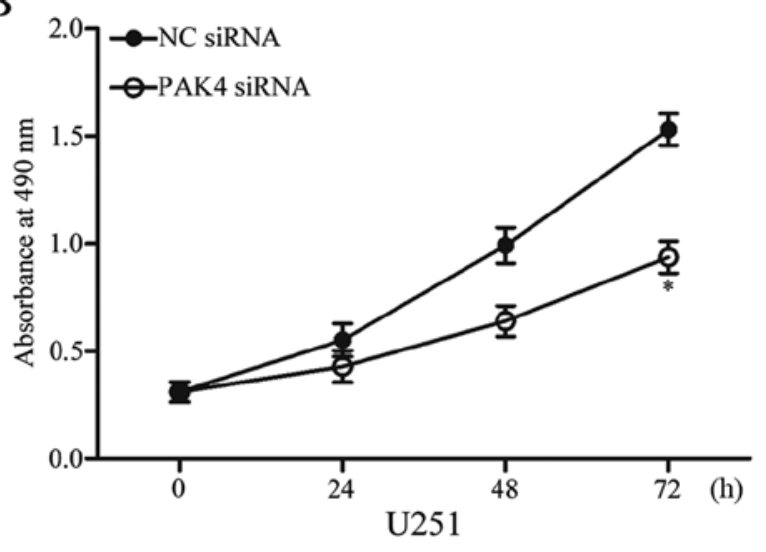

D
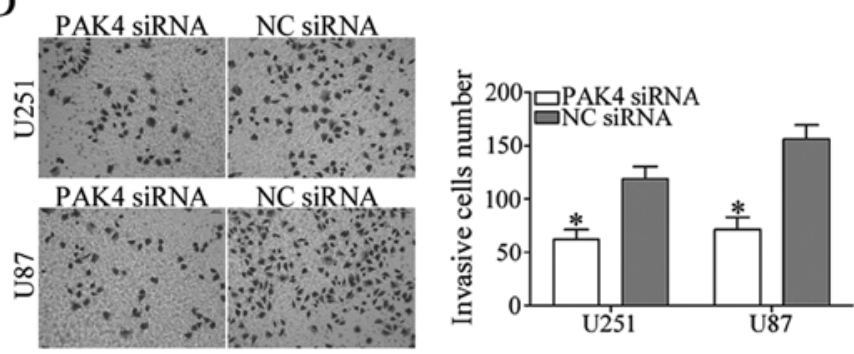

E
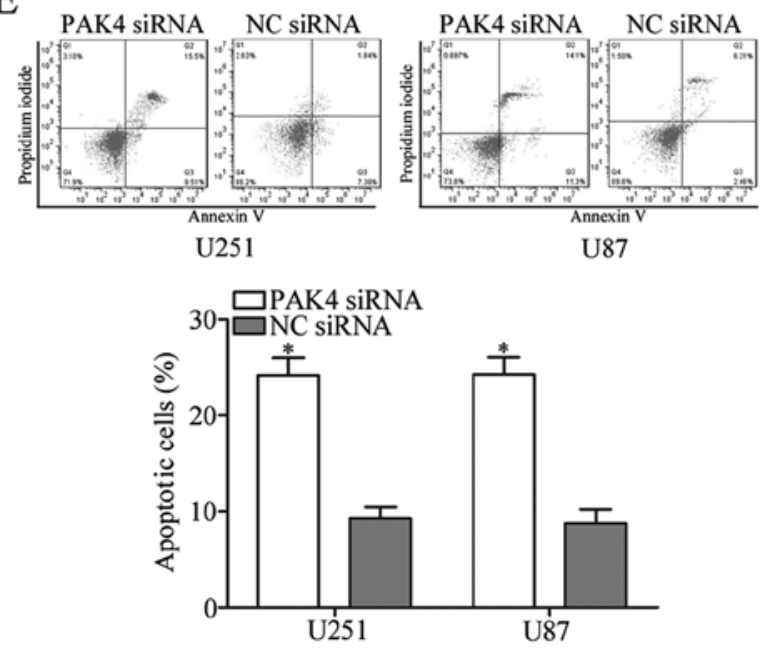

Figure 5. PAK4 knockdown imitates the roles of miR-342 in the U251 and U87 cells. (A) Western blot analysis results showed that the protein level of PAK4 was downregulated in the U251 and U87 cells transfected with PAK4 siRNA. ${ }^{*} \mathrm{P}<0.05$ compared with NC siRNA. (B-E) Downregulation of PAK4 reduced the proliferation and invasion and enhanced apoptosis in the U251 and U87 cells. ${ }^{*} \mathrm{P}<0.05$ compared with NC siRNA.

\section{Discussion}

Glioma has high mortality, high recurrence rate, and low cure rate, because of its rapid growth and metastasis behaviour $(6,7)$. miRNA dysregulation has been implicated in the occurrence and progression of many human cancers, such as bladder cancer (28), gastric cancer (29), hepatocellular carcinoma (30), colorectal cancer (31) and glioma (32). Thus, some specific miRNAs are potential therapeutic targets for cancer diagnosis, therapy and prognosis $(33,34)$. The present study indicated that miR-342 expression was low in both glioma tissues and cell lines. Additionally, reduced expression of miR-342 was 
A
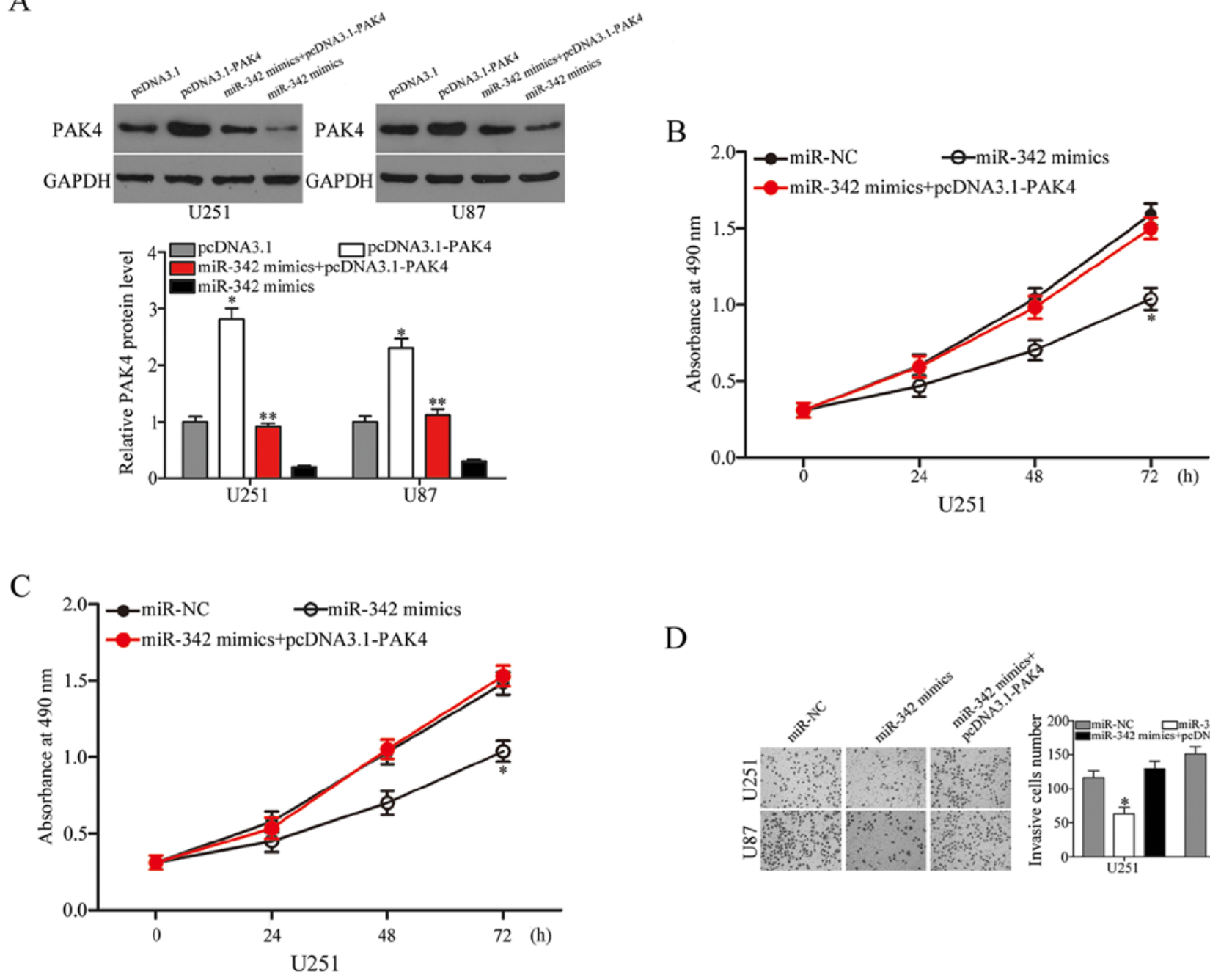

$\mathrm{D}$

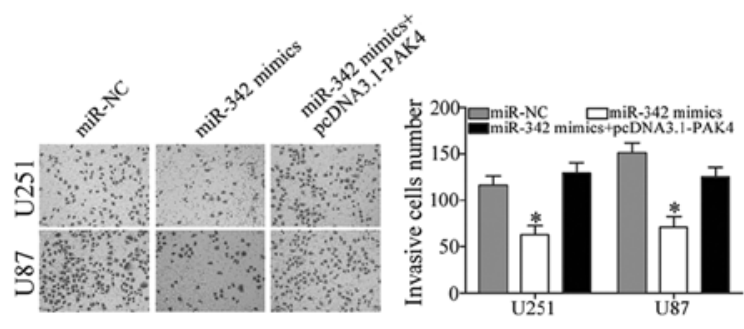

$\mathrm{E}$
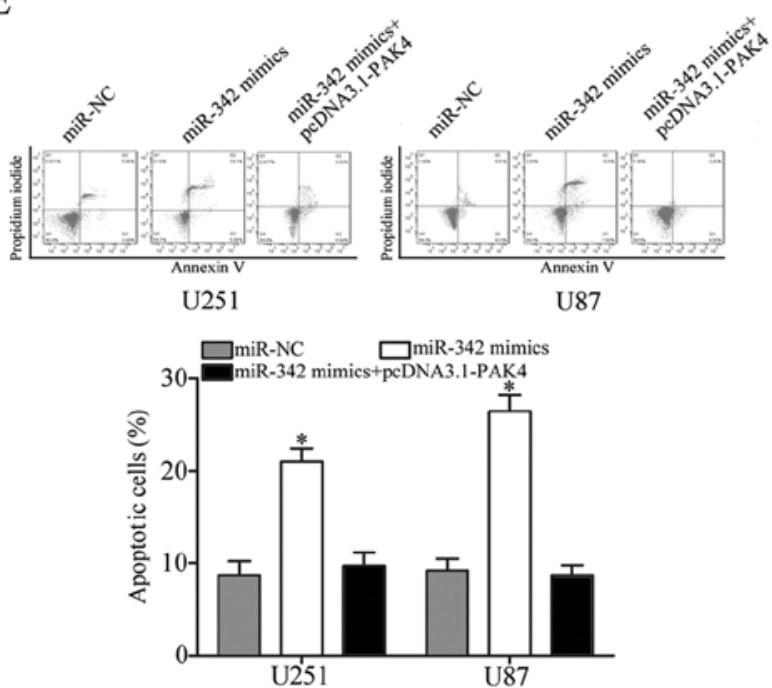

Figure 6. PAK4 overexpression reverses the inhibitory effects of miR-342 in the glioma cells. (A) PAK4 protein expression in U251 and U87 transfected with pcDNA3.1, pcDNA3.1-PAK4, miR-342 mimics or miR-342 mimics+pcDNA3.1-PAK4 was measured by western blot analysis. * $<0.05$ compared with pcDNA3.1. ${ }^{* *} \mathrm{P}<0.05$ compared with miR-342 mimics. (B-E) Cell proliferation, invasion and apoptosis were determined in the U251 and U87 transfected with miR-342 mimics, miR-NC or miR-342 mimics+pcDNA3.1-PAK4. ${ }^{*} \mathrm{P}<0.05$ compared with miR-NC and miR-342 mimics+pcDNA3.1-PAK4.

significantly correlated with advanced WHO grades and low KPS scores of glioma patients. According to these results, we supposed that miR-342 may act as a tumour suppressor in glioma. To confirm this hypothesis, MTT assay, cell invasion 

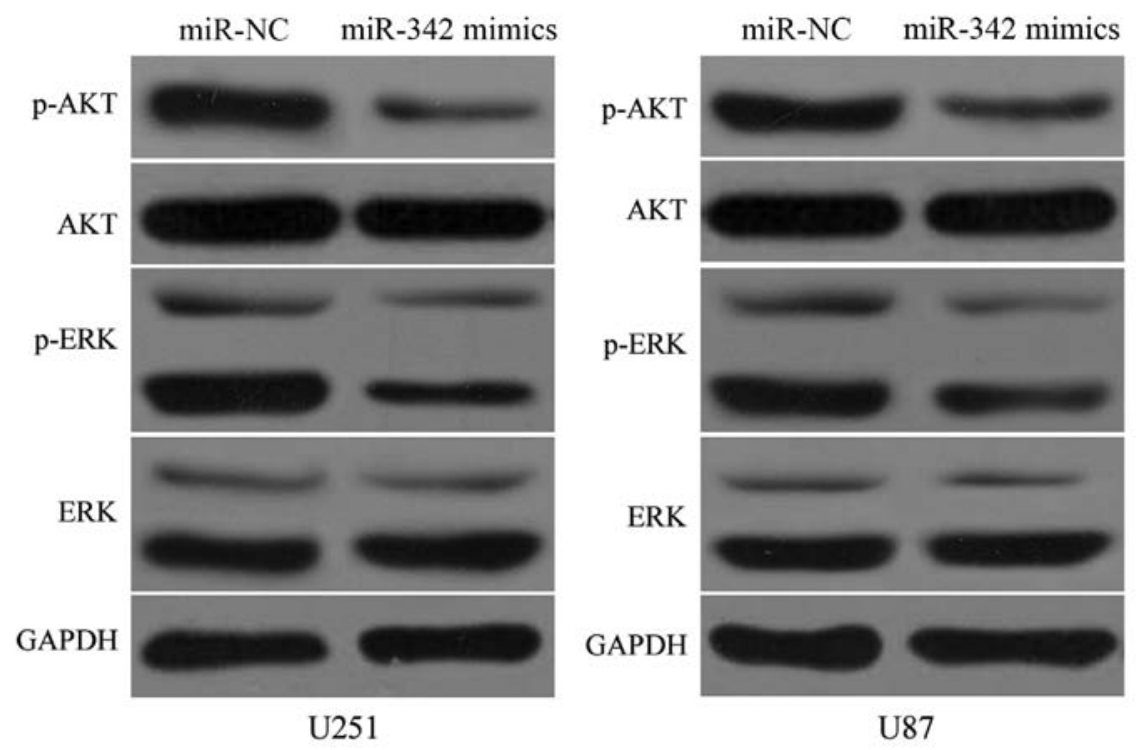

Figure 7. Western blot analysis was conducted to determine the protein levels of p-AKT, p-ERK, AKT and ERK in the U251 and U87 cells transfected with miR-342 mimics or miR-NC.

assay and flow cytometry analysis were performed to examine the effects of miR-342 on cell proliferation and invasion and apoptosis of glioma. We found that the restoration of miR-342 expression suppresses the proliferation and invasion and enhances the apoptotic abilities of glioma cells. Moreover, PAK4 was identified as a direct and functional target of miR-342 in glioma. The upregulation of miR-342 expression inactivated the AKT and ERK pathways in glioma.

miR-342 was previously found to be aberrantly expressed and plays important roles in various human cancers. For example, miR-342 was observed to be downregulated in ER $\alpha$-positive breast cancer tissues and cell lines and significantly correlated with HER2 and VEGF expression status $(20,21)$. miR-342 was found to be decreased in tamoxifen-resistant tumour cells. Enforced expression of miR-342 improved the chemosensitivity of breast cancer cells to tamoxifen (22). Wang et al reported that miR-342 overexpression inhibits colorectal cancer cell growth and metastasis both in vitro and in vivo (23). Zhao and Zhang (35) then revealed that miR-342 upregulation suppresses cell proliferation in hepatocellular carcinoma by regulating the $\mathrm{NF}-\kappa \mathrm{B}$ pathway. A study by Xie et al (36) found that resumption of miR-342 expression can reduce cell proliferation and invasion in vitro and cell growth in vivo in non-small cell lung cancer. Li et al (37) demonstrated that miR-342 re-expression attenuated cervical cancer cell proliferation, migration and invasion. These findings indicated that miR-342 has fundamental roles in carcinogenesis and progression of malignant tumours and illustrated potential of miR-342 as a therapeutic target for various cancers.

Exploring the downstream targets of miR-342 can improve our understanding regarding the underlying molecular mechanisms involved in the tumour-suppressing roles of miR-342 on glioma cells. Notably, several tumour suppressors have been identified as direct targets of miR-342 for example, DNMT1 (23), FOXM1 (38) and FOXQ1 (38) in colorectal cancer; IKK- $\gamma$ (35), TAB2 (35) and TAB3 (35) in hepatocellular carcinoma; RAP2B (36) in non-small cell lung cancer; and FOXM1 (37) in cervical cancer. Through online bioinformatics analysis, PAK4 was predicted to contain a miR-342 seed match at position 488-494 of the PAK4-3'UTR. To test whether miR-342 directly targets 3'UTR of PAK4, we performed luciferase report assays. By measuring changes in the luciferase activity, we demonstrated that miR-342 directly targets 3'UTR of PAK4. Additionally, ectopic expression of miR-342 reduced the PAK4 expression level in glioma cells at the mRNA and protein levels. The expression level of PAK4 was upregulated and negatively correlated with miR-342 expression in glioma tissues. PAK4 knockdown have roles similar to those of miR-342 overexpression in glioma cells, and the upregulation of PAK4 reverses the effects of miR-342 in glioma cells. Moreover, miR-342 can inactivate the AKT and ERK pathways in glioma cells. These results suggested that miR-342 served as a tumour suppressor in glioma, at least in part, by directly targeting PAK4 and indirectly regulating the AKT and ERK pathways.

PAKs belong to a family of serine or threonine kinases, which are best characterized as downstream effectors of Rac and Cdc42 (39). This family comprise 6 mammalian isoforms, and can be divided into two groups: group A, which includes PAKs 1, 2 and 3 and group B, which contains PAKs 4, 5 and $6(40,41)$. PAK4, located at 19q13.2, was reported to be upregulated in multiple human cancers, such as pancreatic cancer (42), colorectal cancer (43), gastric cancer (44) and renal cell carcinoma (45). Increasing evidence indicated that PAK4 plays a significant role in a variety of cellular functions, such as cell proliferation, migration, invasion, apoptosis, actin cytoskeletal changes and cytoskeletal organisation (46). Furthermore, PAK4 was identified to serve important functions in tumourigenesis and tumour development (47-49). In glioma, PAK4 is overexpressed and significantly correlated with pathological grades. PAK4 downregulation repressed glioma cell proliferation, motility and adhesion (25). These data indicate a central role of PAK4 in glioma pathogenesis. 
The findings of the present study identified PAK4 as a direct gene target of miR-342 and suggested that the miR-342/PAK4 pathway is a promising therapeutic target for the treatment of gliomas.

In conclusion, this study showed a significantly low expression level of miR-342 in glioma tissues and cell lines. Our results indicated that miR-342 suppresses tumour in glioma by directly targeting PAK4 and indirectly regulating the AKT and ERK pathways. Thus, miR-342 may be a candidate diagnostic marker of glioma and a potential therapeutic target for the treatment of patients with this disease.

\section{Acknowledgements}

This study was supported by the Zhejiang Provincial Natural Science Foundation (no. LY16H160051); Medical Scientific Research Foundation of Zhejiang Province, China (2012RCA042); Zhejiang Provincial Surgical 'Top Key' Discipline (no. kfjj2011005).

\section{References}

1. Omuro A and DeAngelis LM: Glioblastoma and other malignant gliomas: A clinical review. JAMA 310: 1842-1850, 2013.

2. Vigneswaran K, Neill S and Hadjipanayis CG: Beyond the World Health Organization grading of infiltrating gliomas: Advances in the molecular genetics of glioma classification. Ann Transl Med 3: 95,2015

3. Lou M and Zhao Y: Satisfactory therapy results of combining nimustine with nicardipine against glioma at advanced stage J Cancer Res Ther 11: 1030, 2015.

4. Li BO, Meng C, Zhang X, Cong D, Gao X, Gao W, Ju D and Hu S: Effect of photodynamic therapy combined with torasemide on the expression of matrix metalloproteinase 2 and sodiumpotassium-chloride cotransporter 1 in rat peritumoral edema and glioma. Oncol Lett 11: 2084-2090, 2016.

5. Paw I, Carpenter RC, Watabe K, Debinski W and Lo HW: Mechanisms regulating glioma invasion. Cancer Lett 362: 1-7, 2015.

6. Giese A, Bjerkvig R, Berens ME and Westphal M: Cost of migration: Invasion of malignant gliomas and implications for treatment. J Clin Oncol 21: 1624-1636, 2003.

7. Laks DR, Visnyei K and Kornblum HI: Brain tumor stem cells as therapeutic targets in models of glioma. Yonsei Med J 51: 633-640, 2010.

8. Bartel DP: MicroRNAs: Genomics, biogenesis, mechanism, and function. Cell 116: 281-297, 2004.

9. Valinezhad Orang A, Safaralizadeh R and KazemzadehBavili M: Mechanisms of miRNA-mediated gene regulation from common downregulation to mRNA-specific upregulation Int J Genomics 2014: 970607, 2014.

10. Luo W and Sehgal A: Regulation of circadian behavioral output via a MicroRNA-JAK/STAT circuit. Cell 148: 765-779, 2012.

11. Rachagani S, Macha MA, Menning MS, Dey P, Pai P, Smith LM, Mo YY and Batra SK: Changes in microRNA (miRNA) expression during pancreatic cancer development and progression in a genetically engineered KrasG12D;Pdx1-Cre mouse (KC) model. Oncotarget 6: 40295-40309, 2015.

12. Bagnoli M, Canevari S, Califano D, Losito S, Maio MD, Raspagliesi F, Carcangiu ML, Toffoli G, Cecchin E, Sorio R, et al; Multicentre Italian Trials in Ovarian cancer (MITO) translational group: Development and validation of a microRNA-based signature (MiROvaR) to predict early relapse or progression of epithelial ovarian cancer: A cohort study. Lancet Oncol 17: 1137-1146, 2016

13. Yang L, Wang YL, Liu S, Zhang PP, Chen Z, Liu M and Tang H: miR-181b promotes cell proliferation and reduces apoptosis by repressing the expression of adenylyl cyclase 9 (AC9) in cervical cancer cells. FEBS Lett 588: 124-130, 2014.

14. Chen Y, Gao DY and Huang L: In vivo delivery of miRNAs for cancer therapy: Challenges and strategies. Adv Drug Deliv Rev 81: 128-141, 2015.
15. Zhao Y, Pang D, Wang C, Zhong S and Wang S: MicroRNA-134 modulates glioma cell U251 proliferation and invasion by targeting KRAS and suppressing the ERK pathway. Tumour Biol 37: 11485-11493, 2016

16. Hong L, Ya-Wei L, Hai W, Qiang Z, Jun-Jie L, Huang A, Song-Tao Q and Yun-Tao L: MiR-519a functions as a tumor suppressor in glioma by targeting the oncogenic STAT3 pathway. J Neurooncol 128: 35-45, 2016.

17. Cui R, Guan Y, Sun C, Chen L, Bao Y, Li G, Qiu B, Meng X, Pang C and Wang Y: A tumor-suppressive microRNA, miR-504, inhibits cell proliferation and promotes apoptosis by targeting FOXP1 in human glioma. Cancer Lett 374: 1-11, 2016.

18. Zhang X, Ding H, Han Y, Sun D, Wang H and Zhai XU: The significance of microRNA-184 on JAK2/STAT3 signaling pathway in the formation mechanism of glioblastoma. Oncol Lett 10: 3510-3514, 2015.

19. Li Y, Min D, Wang K, Yin S, Zheng H and Liu L: MicroRNA-152 inhibits cell proliferation, migration and invasion by directly targeting MAFB in nasopharyngeal carcinoma. Mol Med Rep 15: 948-956, 2017.

20. He YJ, Wu JZ, Ji MH, Ma T, Qiao EQ, Ma R and Tang JH: miR-342 is associated with estrogen receptor- $\alpha$ expression and response to tamoxifen in breast cancer. Exp Ther Med 5: 813-818, 2013.

21. Savad S, Mehdipour P, Miryounesi M, Shirkoohi R, Fereidooni F, Mansouri F and Modarressi MH: Expression analysis of MiR-21, MiR-205, and MiR-342 in breast cancer in Iran. Asian Pac J Cancer Prev 13: 873-877, 2012.

22. Cittelly DM, Das PM, Spoelstra NS, Edgerton SM, Richer JK, Thor AD and Jones FE: Downregulation of miR-342 is associated with tamoxifen resistant breast tumors. Mol Cancer 9: 317, 2010.

23. Wang H, Wu J, Meng X, Ying X, Zuo Y, Liu R, Pan Z, Kang T and Huang W: MicroRNA-342 inhibits colorectal cancer cell proliferation and invasion by directly targeting DNA methyltransferase 1. Carcinogenesis 32: 1033-1042, 2011.

24. Livak KJ and Schmittgen TD: Analysis of relative gene expression data using real-time quantitative PCR and the 2 (-Delta Delta C (T)) method. Methods 25: 402-408, 2001.

25. Kesanakurti D, Chetty C, Rajasekhar Maddirela D, Gujrati M and Rao JS: Functional cooperativity by direct interaction between PAK4 and MMP-2 in the regulation of anoikis resistance, migration and invasion in glioma. Cell Death Dis 3: e445, 2012.

26. Tyagi N, Bhardwaj A, Singh AP, McClellan S, Carter JE and Singh S: p-21 activated kinase 4 promotes proliferation and survival of pancreatic cancer cells through AKT- and ERK-dependent activation of NF- $x \mathrm{~B}$ pathway. Oncotarget 5: 8778-8789, 2014

27. Fu X, Feng J, Zeng D, Ding Y, Yu C and Yang B: PAK4 confers cisplatin resistance in gastric cancer cells via PI3K/Akt- and MEK/ERK-dependent pathways. Biosci Rep 34: 34, 2014.

28. Yu G, Jia Z and Dou Z: miR-24-3p regulates bladder cancer cell proliferation, migration, invasion and autophagy by targeting DEDD. Oncol Rep 37: 1123-1131, 2017

29. Lee SW, Park KC, Kim JG, Moon SJ, Kang SB, Lee DS, Sul HJ, Ji JS and Jeong HY: Dysregulation of MicroRNA-196b-5p and MicroRNA-375 in Gastric Cancer. J Gastric Cancer 16: 221-229, 2016.

30. Ma YS, Wu TM, Lv ZW, Lu GX, Cong XL, Xie RT, Yang HQ, Chang ZY, Sun R, Chai L, et al: High expression of miR-105-1 positively correlates with clinical prognosis of hepatocellular carcinoma by targeting oncogene NCOA1. Oncotarget 8 : 11896-11905, 2017.

31. Li Z, Li B, Niu L and Ge L: miR-592 functions as a tumor suppressor in human non-small cell lung cancer by targeting SOX9. Oncol Rep 37: 297-304, 2017.

32. Wei Y, Sun J and Li X: MicroRNA-215 enhances invasion and migration by targeting retinoblastoma tumor suppressor gene 1 in high-grade glioma. Biotechnol Lett 39: 197-205, 2017.

33. Lee KT, Tan JK, Lam AK and Gan SY: MicroRNAs serving as potential biomarkers and therapeutic targets in nasopharyngeal carcinoma: A critical review. Crit Rev Oncol Hematol 103: 1-9, 2016.

34. Li G, Shen Q, Li C, Li D, Chen J and He M: Identification of circulating MicroRNAs as novel potential biomarkers for hepatocellular carcinoma detection: A systematic review and meta-analysis. Clin Transl Oncol 17: 684-693, 2015.

35. Zhao L and Zhang Y: miR-342-3p affects hepatocellular carcinoma cell proliferation via regulating NF- $\kappa B$ pathway. Biochem Biophys Res Commun 457: 370-377, 2015. 
36. Xie X, Liu H, Wang M, Ding F, Xiao H, Hu F, Hu R and Mei J: miR-342-3p targets RAP2B to suppress proliferation and invasion of non-small cell lung cancer cells. Tumour Biol 36: 5031-5038, 2015.

37. Li XR, Chu HJ, Lv T, Wang L, Kong SF and Dai SZ: miR-342-3p suppresses proliferation, migration and invasion by targeting FOXM1 in human cervical cancer. FEBS Lett 588: 3298-3307, 2014.

38. Weng W, Okugawa Y, Toden S, Toiyama Y, Kusunoki M and Goel A: FOXM1 and FOXQ1 are promising prognostic biomarkers and novel targets of tumor-suppressive miR-342 in human colorectal cancer. Clin Cancer Res 22: 4947-4957, 2016.

39. Bokoch GM: Biology of the p21-activated kinases. Annu Rev Biochem 72: 743-781, 2003.

40. Rane CK and Minden A: P21 activated kinases: Structure, regulation, and functions. Small GTPases 5: 5, 2014.

41. Menges CW, Sementino E, Talarchek J, Xu J, Chernoff J, Peterson JR and Testa JR: Group I p21-activated kinases (PAKs) promote tumor cell proliferation and survival through the AKT1 and Raf-MAPK pathways. Mol Cancer Res 10: 1178-1188, 2012.

42. Yeo D, He H, Baldwin GS and Nikfarjam M: The role of p21-activated kinases in pancreatic cancer. Pancreas 44: 363-369, 2015.

43. Song B, Wang W, Zheng Y, Yang J and Xu Z: P21-activated kinase 1 and 4 were associated with colorectal cancer metastasis and infiltration. J Surg Res 196: 130-135, 2015.
44. Kobayashi K, Inokuchi M, Takagi Y, Otsuki S, Fujimori Y, Sato Y, Yanaka Y, Higuchi K, Aburatani T, Tomii C, et al: Prognostic significance of PAK4 expression in gastric cancer. J Clin Pathol 69: 580-585, 2016.

45. Liu W, Yang Y, Liu Y, Liu H, Zhang W, Xu L, Zhu Y and Xu J: p21-Activated kinase 4 predicts early recurrence and poor survival in patients with nonmetastatic clear cell renal cell carcinoma. Urol Oncol 33: 205.e13-21, 2015.

46. Gnad F, Young A, Zhou W, Lyle K, Ong CC, Stokes MP, Silva JC, Belvin M, Friedman LS, Koeppen H, et al: Systems-wide analysis of K-Ras, Cdc42, and PAK4 signaling by quantitative phosphoproteomics. Mol Cell Proteomics 12: 2070-2080, 2013.

47. Dart AE and Wells CM: P21-activated kinase 4 - not just one of the PAK. Eur J Cell Biol 92: 129-138, 2013.

48. Siu MK, Chan HY, Kong DS, Wong ES, Wong OG, Ngan HY Tam KF, Zhang H, Li Z, Chan QK, et al: p21-activated kinase 4 regulates ovarian cancer cell proliferation, migration, and invasion and contributes to poor prognosis in patients. Proc Nat Acad Sci USA 107: 18622-18627, 2010.

49. Ahmed T, Shea K, Masters JR, Jones GE and Wells CM: A PAK4-LIMK1 pathway drives prostate cancer cell migration downstream of HGF. Cell Signal 20: 1320-1328, 2008. 